\title{
Laterality effects of human pudendal nerve stimulation on corticoanal pathways: evidence for functional asymmetry
}

\author{
S Hamdy, P Enck, Q Aziz, S Uengoergil, A Hobson, D G Thompson
}

\begin{abstract}
Background-Although motor and sensory pathways to the human external anal sphincter are bilateral, a unilateral pudendal neuropathy may still disrupt anal continence. Anal continence can, however, be preserved despite unilateral pudendal damage, and so to explain those differing observations, we postulated that pudendal innervation might be asymmetric.

Aims-To explore the individual effects of right and left pudendal nerve stimulation on the corticofugal pathways to the human external anal sphincter and thus assess evidence for functional asymmetric pelvic innervation.

Methods-In eight healthy subjects, anal sphincter electromyographic responses, evoked to transcranial magnetic stimulation of the motor cortex, were recorded 5-500 msec after digital transrectal electrical conditioning stimuli applied to each pudendal nerve.
\end{abstract}

Results-Right or left pudendal nerve stimulation evoked anal responses of similar latencies but asymmetric amplitudes in six subjects: dominant responses $(>50 \%$ contralateral side) from the right pudendal in four subjects and from the left in two. Cortical stimulation also evoked anal responses with amplitude 448 (121) $\mu \mathrm{V}$ and latency 20.9 (1.1) msec. When cortical stimulation was preceded by pudendal nerve stimulation, the cortical responses were facilitated at interstimulus intervals of 5-20 msec. Dominant pudendal nerve stimulation induced greater facilitation of the cortically evoked responses than the non-dominant nerve.

Department of Gastroenterology, Hope Hospital, Eccles Old Road, Manchester M6 8HD, UK

S Hamdy

Q Aziz

A Hobson

D G Thompson

Department of Gastroenterology, Heinrich Heine University, Dusseldorf, Germany

P Enck

$S$ Uengoergil

Correspondence to: Dr Hamdy.

Accepted for publication 25 January 1999

The external anal sphincter (EAS) is an important striated muscle terminating the intestinal tract, which is bilaterally innervated by the somatic fibres of the $2 \mathrm{nd}$, $3 \mathrm{rd}$, and 4 th sacral roots via the pudendal nerves, ${ }^{12}$ and functions to maintain volitional faecal continence. Anal incontinence represents a distressing complication of pelvic nerve injury, for example in association with instrumental deliveries at childbirth; some studies suggest that postpartum incontinence can be explained mainly by direct damage to innervation of the pelvic floor and anal muscles. ${ }^{34}$ In fact, detailed assessment of the anatomy of the incontinent anal sphincter by endoscopic ultrasound, reported in up to $40 \%$ of traumatic deliveries, suggests that rupture of the sphincter itself might be the primary aetiological factor. ${ }^{5}$ It is important to recognise, however, that in some cases, pudendal neuropathy may still be the sole identifiable abnormality. ${ }^{6}$ Indeed, studies using direct stimulation of the pudendal nerve to assess the efferent innervation to the EAS in patients with faecal incontinence ${ }^{7-10}$ have indicated, perhaps surprisingly, that a unilateral pudendal neuropathy is commonly associated with anal sphincter dysfunction and incontinence, despite its bilateral motor innervation. This may indicate that sphincter innervation is asymmetric in many individuals, with one pathway being more dominant than the other.

In previous studies of corticoanal motor function, we have shown that unilateral conditioning stimuli applied to either the lumbosacral spinal roots or pudendal nerves, can facilitate the cortically evoked anal response, a process that was specific to pelvic nerve innervation. ${ }^{11}$ So, while it is clear that central and peripheral neural interactions, via the cerebral cortex and pelvic nerves, are important in the regulation of anal continence, no data exist on the functional relation between the cortex and each pudendal nerve, and consequently interpudendal differences. The aim of our present study was therefore to explore how the cortical pathways to the EAS are influenced by individual pudendal nerve conditioning and thus to determine evidence for pudendal nerve asymmetry in modulating sphincter function.

\section{Methods}

SUBJECTS

Healthy adult, right handed volunteers $(n=8$, seven men and one nulliparous woman; mean age 31 years, range 23-45) were recruited from personnel affiliated with the research units involved in the project. None reported any gastrointestinal, pelvic floor, or anorectal
Abbreviations used in this paper: EAS, external anal sphincter; EMG, electromyographic; ISI, interstimulus interval; T, Tesla. 
problems and all gave informed written consent before study.

\section{MAGNETIC STIMULATION}

Magnetic stimulation of the cerebral cortex was performed using a commercially available magnetic stimulator (Magstim 200, MAGSTIM Company Limited, Whitland, Dyfield, Wales, UK).

\section{Cortical stimulation}

Motor cortex stimulation was performed using a magnetic stimulator connected to a $110 \mathrm{~mm}$ diameter double cone coil (Type 9920, MAGSTIM Company Limited) which, when positioned over the vertex of the cranium, induces currents along the interhemispheric fissure, thereby stimulating the region of the motor cortex innervating pelvic and lower limb musculature. ${ }^{12}$

\section{ELECTRICAL STIMULATION \\ Pudendal nerve stimulation}

Electrical stimulation of each pudendal nerve was performed using the St Marks glove electrode (Model 13L40, Dantec, Tonsbakken, Skovlunde, Denmark). The stimulation electrode pair was positioned over the tip of the operator's index finger, and then inserted intrarectally, to the ischial spine adjacent to the pudendal nerve. ${ }^{8}$ The electrode pair (interelectrode distance $=1.5 \mathrm{~cm}$ ) was connected to an electrical stimulator device (Stimulator Model DS7, Digitimer, Welwyn Garden City, UK) via a trigger generator (Neurolog System, Digitimer) which delivered single stimuli (pulse duration $0.1 \mathrm{msec}$, voltage $280 \mathrm{~V}$ ) at a standard, supramaximal intensity of $15 \mathrm{~mA}$.

Combined pudendal and cortical stimulation This was performed by connecting both the magnetic stimulator and the electrical stimulator to a timing device (Neurolog System, Digitimer), the output of which was programmed to discharge the magnetic stimulator at intervals of 5-500 msec after the electrical stimulus.

ELECTROMYOGRAPHIC RECORDING

Electromyographic (EMG) responses were detected from the striated muscle of the EAS using the St Marks glove electrode. The

Table 1 Individual external anal sphincter EMG response characteristics following right and left pudendal nerve stimulation

\begin{tabular}{lllll}
\hline Subjects $^{*}$ & Sex & Pudendal nerve & Response latency $(\mathrm{ms})$ & Response amplitude $(\mu \mathrm{V})$ \\
\hline 1 & $\mathrm{M}$ & $\mathrm{R}$ & 2.5 & 642 \\
2 & & $\mathrm{~L}$ & 2.3 & 111 \\
& $\mathrm{M}$ & $\mathrm{R}$ & 2.3 & 189 \\
3 & $\mathrm{~L}$ & 2.0 & 100 \\
& $\mathrm{R}$ & $\mathrm{R}$ & 2.2 & 341 \\
& & $\mathrm{~L}$ & 2.0 & 168 \\
5 & $\mathrm{M}$ & $\mathrm{R}$ & 2.0 & 189 \\
& $\mathrm{~L}$ & 2.0 & 81 \\
6 & $\mathrm{R}$ & 2.0 & 137 \\
7 & $\mathrm{M}$ & $\mathrm{L}$ & 1.8 & 373 \\
& $\mathrm{R}$ & 1.8 & 67 \\
8 & $\mathrm{~F}$ & $\mathrm{R}$ & 1.8 & 1197 \\
& $\mathrm{R}$ & 1.5 & 1211 \\
& $\mathrm{~L}$ & $\mathrm{R}$ & 1.8 & 437 \\
& & $\mathrm{~L}$ & 2.3 & 367 \\
\hline
\end{tabular}

${ }^{\star}$ Subjects $1-6$ were considered to have asymmetric pudendal nerve responses, and subjects 7 and 8 symmetric responses. recording electrode pair (interelectrode distance $=1 \mathrm{~cm}$ ), was positioned over the base of the operator's index finger, which when inserted intrarectally, reached the level of EAS.

The electrode pair was connected to a preamplifier (CED 1902, Cambridge Electronic Design, Cambridge, UK) with filter settings of 5-2000 Hz. Response signals were then collected through a laboratory interface (CED 1401 plus, Cambridge Electronic Design) at a sampling rate of $4-8 \mathrm{kHz}$ and fed into a $486 \mathrm{Sx}$ desktop computer for immediate display, data collection, and averaging. During each study, electrode contact was monitored at 10 minute intervals by observing the real time EMG responses to voluntary anal sphincter contractions.

\section{EXPERIMENTAL PROTOCOL}

The protocol described below was presented to, and approved by, the Salford Health Authority Ethics Committee. Throughout each study, the volunteer, having previously emptied his or her rectum by defaecation, lay comfortably on a couch, in either the left or right lateral position, and the vertex of the cranium was identified according to the international 10-20 system. ${ }^{13}$ Then, following a digital examination to ensure that the rectum was empty, the St Marks glove electrode was inserted.

Subjects were investigated on each side, in either the left or right lateral position as appropriate. Firstly, in each subject, either the right or the left pudendal nerve was electrically stimulated, at the supramaximal intensity of 15 $\mathrm{mA}$, the order being randomised between individuals. To ensure that each nerve was being optimally stimulated, the orientation of the stimulation electrode was adjusted until the point which produced the shortest latency and largest amplitude response was found. Three stimulations, 15 seconds apart, were then delivered, and the EMG responses recorded. The procedure was repeated for the other pudendal nerve, after the subject had altered position.

Secondly, the cerebral cortex of each subject was stimulated, initially at a discharge intensity of 0.6 Tesla (T), and then increased by intensities of $0.1 \mathrm{~T}$ until anal sphincter EMG responses were obtained, this being defined as the threshold intensity. Three stimuli, 15 seconds apart, were then delivered at $0.4 \mathrm{~T}$ above the threshold stimulus intensity and the EMG responses to each were recorded.

Finally, a series of stimuli were delivered to the right and left pudendal nerves in random order, at the intensities and positions defined above. Each stimulus was followed by stimulation of the cortex, at $0.4 \mathrm{~T}$ above threshold intensity, at intervals of $5,10,20,30,50,100$, 200, 300, and $500 \mathrm{msec}$. Three stimulations, each 15 seconds apart, were delivered for each interval, and the anal sphincter EMG responses recorded. 

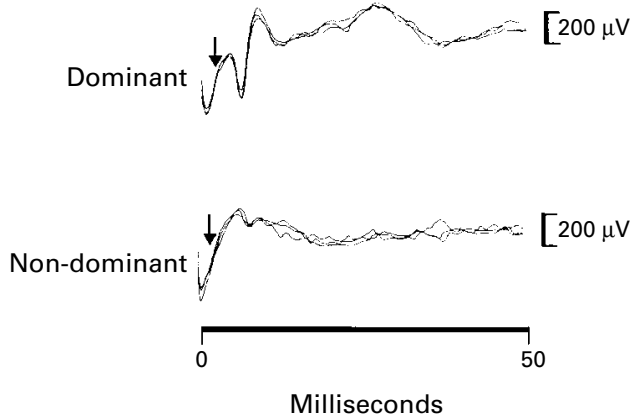

Figure 1 External anal sphincter EMG responses are shown following right (dominant) and left (non-dominant) pudendal nerve stimulation in one individual. Three traces are superimposed to show reproducibility. Stimulus at $0 \mathrm{~ms}$; arrows indicate the onset of the EMG response. The response amplitude from the dominant pudendal nerve is larger than from the non-dominant nerve, despite similar latencies.

DEFINITION OF TERMS

Response latency - the interval between the onset of the stimulus and the onset of the EMG response, expressed in msec.

Response amplitude - the maximum peak to peak voltage of the EMG response, expressed in $\mu \mathrm{V}$.

Response facilitation - enhancement of the EMG response by either a reduction in the response latency or an increase in the response amplitude.
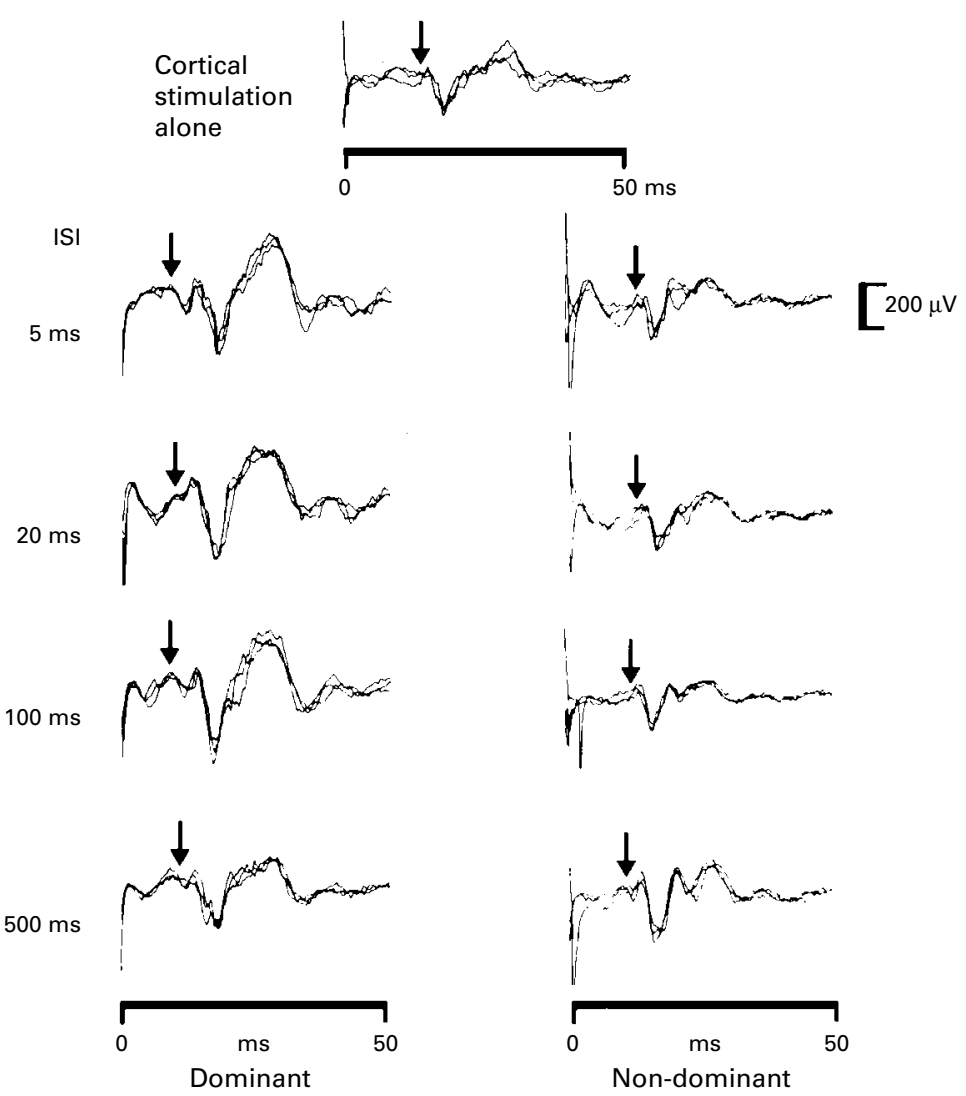

Figure 2 Cortically evoked external anal sphincter EMG responses from one individual following cortical stimulation alone and following dominant and non-dominant pudendal nerve stimulation at different ISIs. Three traces are superimposed to show reproducibility. Cortical stimulus at 0 msec; arrows indicate the onset of the EMG response. Following dominant pudendal nerve stimulation, the cortically evoked responses are facilitated to a much greater degree than with non-dominant pudendal stimulation.
Pudendal response asymmetry - the intraindividual response amplitudes to right and left pudendal nerve stimulation were considered asymmetric if one side exceeded the other by at least $50 \%$. If asymmetry, thus defined, was found, the side with the larger amplitude was referred to as the dominant pudendal nerve and that with the smaller amplitude as the non-dominant pudendal nerve.

\section{DATA ANALYSIS}

For each study, the mean value of the three EMG responses evoked was calculated and used for analysis. Individual anal EMG response amplitudes evoked following right and left pudendal nerve stimulation were first measured. In subjects where the pudendal evoked response amplitudes were asymmetric, the group mean cortically evoked anal EMG responses following dominant pudendal nerve stimulation, across all interstimulus intervals (ISIs), were compared with those following non-dominant nerve stimulation. In subjects where the pudendal evoked response amplitudes were symmetric, the group mean cortically evoked anal EMG responses following right pudendal nerve stimulation, across all ISIs, were compared with those following left pudendal nerve stimulation.

STATISTICAL TESTS

The normality of the data was first assessed using the Shapiro-Wilks test. ${ }^{14}$ This showed that the correlations obtained for the pudendal and cortical response latencies were consistent with normality; the paired, two tailed, Student's $t$ test was therefore applied for intrasubject comparisons. By contrast, the correlations obtained for the pudendal and cortical response amplitudes were not consistent with normality, and so natural logarithms of the data were calculated and the Shapiro-Wilks test reapplied. This showed that the correlations obtained for both the transformed pudendal and cortical response amplitudes remained inconsistent with normality, so the Wilcoxon signed rank sum test was used on the untransformed data. Results are expressed in the text as mean (SEM) unless stated otherwise. A p value of 0.05 or less was taken to indicate that any observed differences were unlikely to have occurred by chance.

\section{Results}

EAS RESPONSES TO INDIVIDUAL PUDENDAL NERVE STIMULATION

In all subjects, stimulation of either the right or left pudendal nerves always evoked anal sphincter EMG responses. While intraindividual and interindividual response latencies were similar between nerves (right: $2.2(0.2)$, left: $2.1(0.1) \mathrm{msec})$, response amplitudes varied (table 1, fig 1). The pudendal responses were found to be asymmetric in six subjects, the right nerve being dominant in four subjects and the left in two subjects. In the remaining two subjects the responses were symmetric. 
- Non-dominant pudendal nerve stimulation

- Dominant pudendal nerve stimulation

- Symmetric left pudendal nerve stimulation

- Symmetric right pudendal nerve stimulation
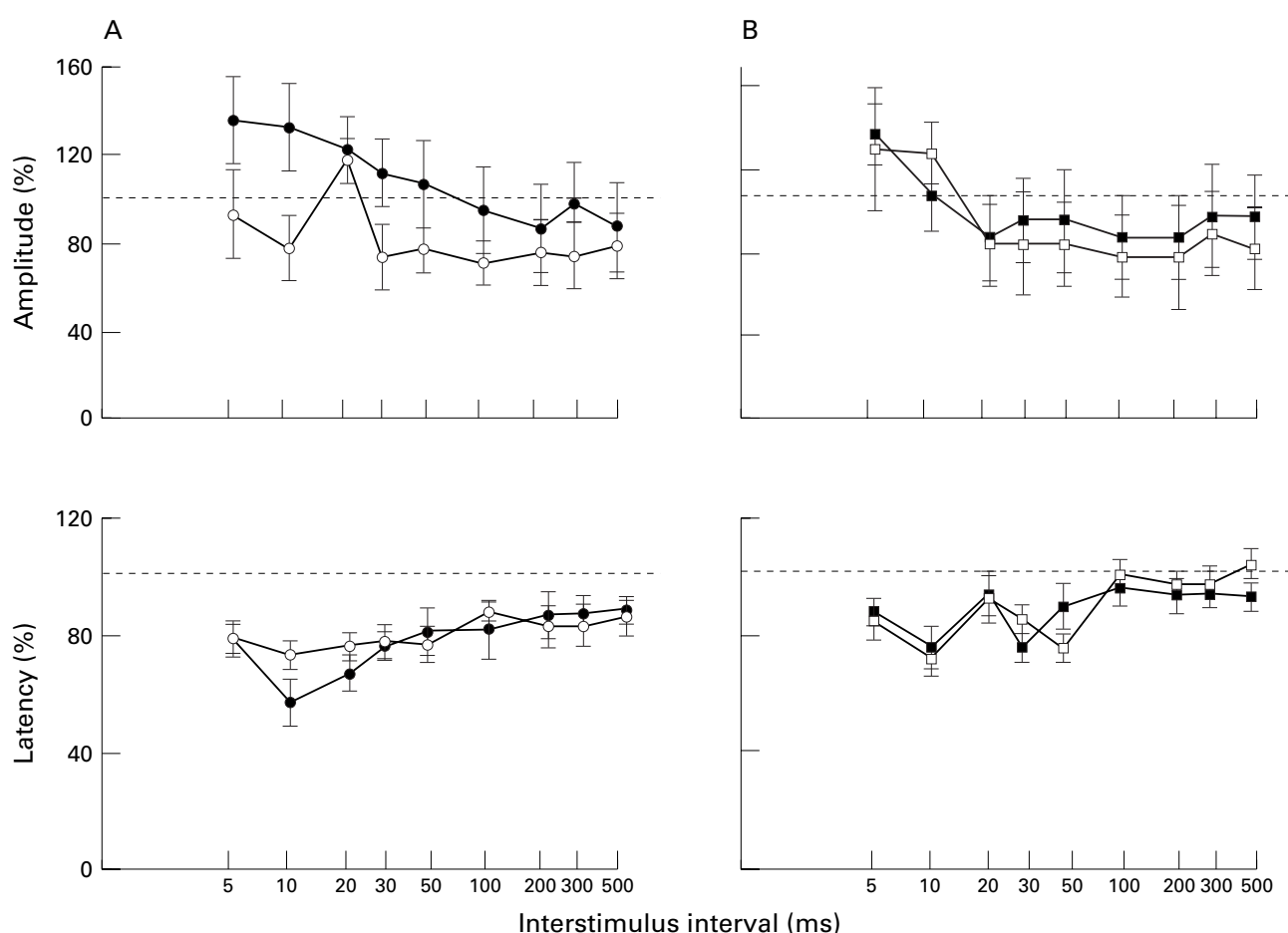

Figure 3 Effects of: $(A)$ dominant (closed circle) and non-dominant pudendal nerve (open circle) stimulation ( $n=6)$; and (B) symmetric right (closed square) and left (open square) pudendal nerve stimulation $(n=2)$ on the amplitudes and latencies of the cortically evoked anal responses at increasing ISIs. The vertical axes show the percentage of the response to cortical stimulation alone; the horizontal broken line indicates $100 \%$. Dominant pudendal nerve stimulation produces relatively greater facilitation of the cortically evoked responses than non-dominant pudendal nerve stimulation $(p<0.001)$; in contrast, prior symmetrical pudendal nerve stimulation has equal effects from either side.

EAS RESPONSES TO CORTICAL STIMULATION Cortical stimulation (mean threshold intensity $1.4(0.1) \mathrm{T}$ ) always evoked biphasic or triphasic responses in the anal sphincter (fig 2) with an amplitude of 448 (121) $\mu \mathrm{V}$ and a latency of 22.1 (1.0) msec.

COMPARISON OF THE EFFECTS OF EACH PUDENDAL NERVE STIMULATION OF THE CORTICALLY EVOKED EAS RESPONSES

When either right or left pudendal nerve stimulation preceded cortical stimulation, the cortically evoked anal responses were facilitated, the effect appearing maximal for both response amplitude and/or latency at intervals of 5-20 msec (figs 2 and 3). However, when individual interpudendal effects were compared, subjects with asymmetric pudendal responses showed greater amplitude facilitation from stimulation of the dominant pudendal nerve, than from the non-dominant nerve $(\mathrm{p}<0.001$; fig 3$)$. Those subjects with symmetric pudendal responses, however, showed no difference between each side in facilitation of the cortically evoked responses.

\section{Discussion}

Anal continence represents an important physiological and socially essential gastrointestinal function which is regulated by sensorimotor interactions within the anal sphincter and pelvic floor. ${ }^{15}$ In particular, contraction of the EAS serves to increase anal canal pressure, both voluntarily, as when the urge to defaecate becomes strong, and via more involuntary reflexes, for instance during coughing, when intra-abdominal pressure suddenly rises. ${ }^{12}$ In either case, the cerebral cortex is able to modulate this activity via powerful descending input to the pelvic plexus and sacral nerves, so that defaecation can be resisted until a socially convenient opportunity arises. The importance of this higher centre control of continence is well recognised, disruption at any site along the pathway from cortex to sphincter muscle leading to EAS dysfunction and potential incontinence; this is seen in conditions such as stroke, ${ }^{16}$ multiple sclerosis, ${ }^{17}$ and spinal or pudendal nerve injury. ${ }^{4}$

More controversial is the issue of the causal role of pudendal neuropathies in the aetiology of anal incontinence. For example, pudendal neuropathy may be commonly observed in idiopathic incontinence, ${ }^{9} 1819$ which may be bilateral, but, in many cases, can be unilateral. Furthermore, a number of studies have shown clear abnormalities in pudendal nerve function (as determined by prolonged nerve conduction latencies) in incontinent female patients following vaginal delivery both with and without instrumental intervention. ${ }^{3420}$ However, the more recent development of endoscopic anal ultrasound for assessing sphincter integrity, has shown that the major proportion of these 
patients previously labelled as having idiopathic or neurogenic incontinence secondary to pelvic innervation damage, also have underlying anatomical defects of the external sphincter. ${ }^{5}$ Indeed, a successful surgical repair of the EAS does not appear to depend on pudendal function assessed by conduction studies, ${ }^{18}$ suggesting that neurogenic anal incontinence is probably multifactorial and that anatomical defects should always be sought even in the presence of strong evidence implicating pudendal damage. Nonetheless, the electrophysiological assessment of pudendal function remains of some importance, as this parameter may be clinically relevant in differentiating patients with faecal leakage from solid stool incontinence, ${ }^{21}$ and there exist patients without external anal sphincter defects, who have significant continence problems and whose only demonstrable abnormality is that of pudendal neuropathy. ${ }^{6}$

Anatomical and electrophysiological studies of the innervation of the EAS in animals have shown that most of the pudendal projections to and from the EAS are centrally organised in the spinal segments L6 to S3, with the majority being within the S1-S2 segments. ${ }^{22-24}$ Furthermore, the motoneurones innervating the EAS appear to be located in the dorsomedial and ventromedial divisions of Onuf's nucleus in the ventral horn of the spinal cord, while the afferent axonal projections appear to cluster within the marginal zone, intermediate grey, and dorsal grey surrounding the nucleus gracile of the dorsal column and lamina I around the dorsal horn of the spinal cord. ${ }^{22}$ Of interest, is the observation that while there is clear ipsilateral spinal predominance of these projections during retrograde tracing studies of a single nerve, in both the efferent and afferent pathways, there is contralateral axonal connectivity across the midline, suggesting degrees of bilateral convergence. ${ }^{22-24}$ Indeed, this connectivity appears to be present even in the EAS muscle itself. ${ }^{25}$ While the physiological significance of this observation remains to be determined, it could be speculated that such convergence may be important in reorganisation of spinal and pudendal innervation of the EAS after central and peripheral nerve damage.

Our study has now shown that the cortical pathways to the human EAS are modulated asymmetrically by conditioning stimuli applied to the pudendal nerves. As with our previous studies, ${ }^{11}$ the effect was facilitatory, and was best observed at intervals of $5-20 \mathrm{msec}$ after the pudendal stimulus, suggesting that antidromic excitation of the sacral motoneurones had been induced with a time course of increased excitability lasting in the range of $20 \mathrm{msec}$. However, when individual pudendal nerve responses were examined, clear asymmetries in the size of the sphincter responses were observed, despite identical stimulation parameters. Furthermore, in those subjects with asymmetric responses, prior stimulation of the dominant pudendal nerve induced notably better facilitation of the corticoanal pathways than the non-dominant nerve. While it is possible to suggest that the differences in pudendal responsiveness relate to suboptimal excitation of the non-dominant nerve, this is unlikely as we found that the response latencies between the two sides were similar, compatible with equivalent nerve stimulation. Furthermore, careful local adjustments of the stimulating electrode were performed (on each side) in order to obtain the greatest and most reproducible evoked response. It is therefore more probable that the difference between the two sides, both in pudendal response amplitude and induced corticoanal facilitation, reflects a true difference in the functional motor (and possibly sensory) innervation of the EAS, and that in many individuals, one pudendal nerve is dominant. In support of this concept, asymmetries in human pudendal nerve afferent fibres have been described during intraoperative monitoring of the dorsal sacral roots following dorsal penile or clitoral nerve stimulation $^{26}$ and animal data have also shown that the pudendal motor innervation to the urethral sphincter can be asymmetric. ${ }^{27}$

An important question arising from our findings, is at what level in the innervation of the EAS does the asymmetry originate? For example, anatomical studies in monkeys have indicated that there may be substantial overlap across the midline in the pudendal innervation of the EAS muscles on the two sides, a consequence of interdigitation of muscle fascicles. ${ }^{25}$ As a result, it may be argued that the interpudendal asymmetry observed in our study may reflect some underlying lateralised crossover of nerve fibres at the muscular level. Alternatively, a higher level asymmetric input may be driving this anatomical predominance within the muscle. In support of the latter suggestion, it has been shown that the cortical motor and sensory representation of the EAS may be lateralised to one hemisphere or the other, ${ }^{28}{ }^{29}$ which might imply that the lower level circuitry controlling EAS is influenced by lateralisation at the higher level. In this respect, it would be interesting to study both levels in the system to identify whether there is indeed a directional relation between asymmetries in both pudendal and cortical projections to the EAS.

Given the presence of interpudendal asymmetry, it is possible that unilateral damage to the dominant pudendal nerve may predispose individuals to anal dysfunction while damage to the non-dominant nerve may favour preserved anal function. Of interest, anatomical asymmetries, both within and external to the central nervous system, have been suggested to be more pronounced in men compared with women (and in right handers compared with left handers), a possible consequence of androgenic hormonal exposure during early development. ${ }^{30} 31$ In terms of pudendal nerve morphology, sex differences have been described in animals, usually relating to the size and fibre density of the nerve, being greater in males. ${ }^{32}$ While the number of subjects included in our study make it impossible to draw any firm conclusions on differential patterns of male/female pudendal innervation to the anal 
sphincter, it might be that females could be more bilaterally innervated and consequently less likely to suffer from unilateral damage. In this regard, it is interesting to note that the only woman included in our study was one of the symmetrical pudendal responders. Perhaps future clinical studies, comparing the degree of corticoanal facilitation to pudendal nerve stimulation in patients with unilateral pudendal neuropathy, with and without anal incontinence, may provide greater insight into whether pudendal innervation asymmetries contribute to the pathophysiology of faecal incontinence after unilateral nerve damage.

In conclusion, therefore, pudendal nerve stimulation facilitates human cortical motor pathways to the EAS with intraindividual asymmetry, a finding which provides one possible explanation for the variation and degree of anal incontinence following unilateral pudendal nerve injury. The authors thank Ms Josephine Barlow and Mrs Barbara
Unsworth in the Gastrointestinal Physiology Laboratory at Unsworth in the Gastrointestinal Physiology Laboratory at Hope Hospital for their assistance. This work was supported by
grants from the British Digestive Foundation, the Deutsche grants from the British Digestive Foundation, the Deutsche Drschungsgemeinschaft (En 50/10), and the British Council. Dr S Hamdy is a Clinical Trainin
Research Council, United Kingdom.

1 Matzel KE, Schmidt RA, Tanagho EA. Neuroanatomy of the striated muscular anal continence mechanisms. Implications for the use of neurostimulation. Dis Colon Rectum 1990;33:666-73.

2 Bishop B, Garry RC, Roberts TDM, et al. Control of the external sphincter of the anus in the cat. $f$ Physiol 1956;134:229-40.

3 Snooks SJ, Setchell M, Swash M, et al. Injury to innervation of pelvic floor musculature in childbirth. Lancet 1984;ii: 546-50.

4 Tetzschner T, Sorensen M, Rasmussen OO, et al. Pudendal nerve damage increases the risk of fecal incontinence in women with anal sphincter rupture after childbirth. Acto Obstet Gynecol Scand 1995;74:434-40.

5 Sultan AH, Kamm MA, Hudson CN, et al. Anal sphincter disruption during vaginal delivery. N Engl f Med 1993;329: 1905-11.

6 Rieger NA, Sarre RG, Sccone GT, et al. Correlation of pudendal nerve terminal motor latency with the results of pudendal nerve terminal motor latency with the result

7 Kiff ES, Swash M. Normal proximal and delayed distal conKiff ES, Swash M. Normal proximal and delayed distal con-
duction in the pudendal nerves of patients with idiopathic duction in the pudendal nerves of patients with idiopathic
(neurogenic) faecal incontinence. $f$ Neurol Neurosurg (neurogenic) faecal inco

8 Kiff ES, Swash M. Slowed conduction in the pudendal nerves in idiopathic (neurogenic) faecal incontinence. $\mathrm{Br} f$ Surg 1984;71:614-16

9 Sangwan YP, Coller JA, Barrett RC, et al. Unilateral pudendal neuropathy. Significance and implications. Dis Colon Rectum 1996;39:249-51.
10 Snooks SJ, Barnes PR, Swash M. Damage to the innervation of the voluntary anal and periurethral sphincter musculature in incontinence: an electrophysiological study. 7 Neurol Neurosurg Psychiatry 1984;47:1269-73.

11 Hamdy S, Enck P, Aziz Q, et al. Spinal and pudendal nerve modulation of human cortico-anal motor pathways. Am $\mathcal{F}$ Physiol 1998;274:G419-23.

12 Herdmann J, Enck P, Zacchi-Deutschbein P, et al. Speed and pressure characteristics of external anal sphincter contractions. Am F Physiol 1995;32:G225-31.

13 Jasper HH. The 10-20 electrode system of the International Federation. Electroencephalogr Clin Neurophysiol 1958;10: $371-5$.

14 Altman DG. Comparing groups: continuous data. In: DG Altman, ed. Practical statistics for medical research. London: Altman, ed. Practical statistics for medic

15 Christensen J. Motility of the colon. In: LR Johnson, ed. Physiology of the gastrointestinal tract. Vol 1. New York: Raven Press, 1983:445-72.

16 Brocklehurst JC, Andrews K, Richards B, et al. Incidence and correlates of incontinence in stroke patients. f $\mathrm{Am}$ Geriatr Soc 1985;33:540-2.

17 Hinds JP, Eidelman BH, Wald A. Prevalence of bowel dysfunction in multiple sclerosis. Gastroenterology 1990;98: $1538-42$.

18 Sangwan YP, Coller JA, Barrett RC, et al. Unilateral pudendal neuropathy. Impact on outcome of anal sphincter dal neuropathy. Impact on outcome

19 Swash M, Snooks SJ. Nerve stimulation techniques. A Pudendal nerve terminal motor latency, and spinal stimulation. In: MM Henry, M Swash, eds. Coloprotology and the pelvic floor. London: Butterworths, 1985:112-24.

20 Tetzschner T, Sorensen M, Jonsson L, et al. Delivery and pudendal nerve function. Acta Obstet Gynecol Scand 1997;76:324-31.

21 Kafna NJ, Coller JA, Barrett RC, et al. Pudendal neuropathy is the only parameter differentiating leakage from solid stool incontinence. Dis Colon Rectum 1997;40:1220-7.

22 Thor KB, Morgan C, Nadelhaft I, et al. Organization of afferent and efferent pathways in the pudendal nerve of the female cat. F Comp Neurol 1989;288:263-79.

23 Ueyama T, Mizuno N, Takahashi O, et al. Central distribution of efferent and afferent components of the pudendal nerve in macaque monkeys. F Comp Neurol 1985;232:54856.

24 Ueyama T, Arakawa H, Mizuno N. Central distribution of efferent and afferent components of the pudendal nerve in the rat. Anat Embryol 1987;177:37-49.

25 Wunderlich $M$, Swash $M$. The overlapping innervation of the two sides of the external anal sphincter by the pudendal nerves. F Neurol Sci 1983;59:97-109.

26 Deletis V, Vodusek DB, Abbott R, et al. Intra-operative monitoring of the dorsal sacral roots: minimizing the risk of atrogenic micturition disorders. Neurosurery 1992;30:72-

27 Morita T, Kizu N, Kondo S, et al. Ipsilaterality of motor innervation of the canine urethral sphincter. Urol Int 1988; 43:149-56.

28 Turnbull GK, Aziz Q, Hamdy S, et al. Representation of the anal sphincter on the human cerebral cortex [abstract]. Gut 1994;35:S30.

29 Stottrop K, Schnitzler A, Witte OW, et al. Cortical representation of the anal canal [abstract]. Gastroenterology 1998; 114:A843

30 Bishop DVM. Handedness and developmental disorders. Oxford: MacKeith Press, 1990:27-35.

31 Bradshaw JL, Nettleton NC. Human cerebral asymmetry. New Jersey: Prentice-Hall Inc., 1983:21-30.

32 McKenna KE, Nadelhaft I. The organization of the pudendal nerve in the male and female rat. F Comp Neurol 1986; 248:532-49. 